\title{
I-DOPA stimulates the dopaminergic phenotype in human retina
}

Authors: Bojana Radojevic, M.S. ${ }^{1}$, Margarita Mauro-Herrera, Ph.D. ${ }^{1}$, Lea D. Bennett, Ph.D. ${ }^{1,2}$

Author affiliations: ${ }^{1}$ Department of Ophthalmology, University of Oklahoma Health Sciences Center; ${ }^{2}$ Dean McGee Eye Institute, Oklahoma City, OK

Abstract Retinal organoids derived from inducible pluripotent stem cells were used to gain insight into the role of I-DOPA during human retinal development. Dopaminergic gene expression was indicated by assessing two dopamine receptors (DRD1 and DRD2), DOPA decarboxylase (DDC), and tyrosine hydroxylase $(T H)$ via quantitative reverse transcription-polymerase chain reaction at various developmental stages. TH transcript levels started to express around day (D) 42, reached maximal expression D63 and then decreased thereafter. At D29, proliferating retinal progenitors expressed $D R D 1, D R D 2$, and $D D C$ at various levels of mRNA throughout the day. In the presence of I-DOPA, D29 retinal organoids expressed DRD1 but DRD2 mRNA expression was suppressed. Additionally, I-DOPA upregulated TH mRNA prior to dopaminergic amacrine cell (DAC) development. After the appearance of DACs, I-DOPA phase shifted expression of DRD2 and synchronized mRNA expression of $D D C, D R D 2$, and $T H$. The present results suggest unique mechanisms for DA signaling at different stages of development in the human retina. 


\section{Introduction}

Dopamine (DA) is a critical neurotransmitter that integrates signaling in the retina to mediate physiological functions such as circadian entrainment, neurogenesis, photoreceptor disk shedding, intraocular pressure, and refractive development. ${ }^{1-4}$ DA acts through receptors to activate or inhibit distinct pathways thereby mitigating various functions during neurogenesis. In this way, neuronal excitability occurs by second messenger cascades coupled to DA receptors (DRs). DRs are g-protein coupled receptors (GPCRs), classified by the g-protein that they couple to: D1-"like" couple to Gas and D2-like couple to Gai. ${ }^{5-7}$ Additionally, DA is a zeitgeber, or time setter that regulates the expression of circadian rhythm genes and is critically involved in mediating the core molecular clock throughout the body. In adults, disruption of the DA pathway is associated with visual deficits in Parkinsonians disorders and schizophrenia. ${ }^{8}$ During embryogenesis, dysfunction in DA signaling has been linked to myopia, ${ }^{4}$ and ocular albinism ${ }^{9}$.

Research has revealed various processes in neurogenesis that are linked to DA signaling but these events depend on cell type and stage of development. For instance, DA increases neuronal proliferation when D2-like receptors are activated, but D1-like receptor activation decreases proliferation. ${ }^{10}$ Neurons that are GABAergic will migrate when DA binds D1-like receptors but this migration is inhibited by a D2-like receptor agonist. ${ }^{11}$ In cerebral cortical neurons, DA promotes axon growth by D2-like receptor activation which is inhibited with D1-like receptor stimulation. ${ }^{12,13}$ In striatal neurons, the opposite occurs; neurite outgrowth is stimulated by DA binding to D1-like receptors, but not with D2-like receptor activation. ${ }^{14}$ The basis for these effects in neural tissues of rodents is not fully understood and even less is known about DA mechanisms within developing human retinal neurons. The changing roles of this neuromodulator during development may be a fine-tuning mechanism to ensure cell migration and correct placement within the retina to achieve lamination.

DA synthesis occurs in a 2 step reaction whereby tyrosine hydroxylase $(\mathrm{TH})$ converts tyrosine to IDOPA. Next, DOPA decarboxylase (DDC) acts on I-DOPA to form DA (Fig. 1). DA is released and binds to its receptors on the post-synaptic neuron (Fig. 1). In the retina, seminal experiments using chicks have determined that early in embryogenesis extrinsic I-DOPA is made and secreted from the retinal pigmented epithelium (RPE). ${ }^{15}$ This upregulates DA synthesis and occurs prior to the appearance of dopaminergic amacrine cells (DACs). Additionally, at later stages during chick embryogenesis, DA inhibits axonal growth cones $^{16}$ and limits the total number of amacrine cells that can synthesize DA. ${ }^{17}$ Taken together, these studies 
suggest that DA has different molecular affects that depend on cell type, developmental stage, and the local environment.

In the present study, gene expression of $D R D 1, D R D 2, D D C$, and $T H$ define the dopaminergic phenotype. Here we investigate the development of the dopaminergic phenotype and gain insight into the role of I-DOPA using human retinal tissue derived from human inducible pluripotent stem (iPS) cells. These results show that prior to the appearance of DACs, I-DOPA upregulates mRNA expression of TH and DRD1 but suppresses DRD2. After the appearance of DACs, I-DOPA stimulates a phase shift so that DRD2 is expressed maximally and synchronized with mRNA transcription of $D D C$ and $T H$. Altogether, these results suggest unique mechanisms for DA signaling at different stages of development in the human retina.

\section{Methods}

\section{Differentiation protocol}

Human iPS cells were maintained in 6-well plates on Matrigel (Corning). We used two different iPS cell lines GM23270 and GM25256 (Coriel Institute). StemFlex (Thermo Fisher) media was used to maintain the health and pluripotency of the iPS cells and ReLeSR (STEMCELL Technologies) was used for passaging. Differentiation experiments were initiated using an established protocol. ${ }^{18,19}$ Briefly, embryoid body (EB) formation began with transition into neural induction medium [NIM; DMEM:F12 1:1, 1\% N2 supplement, 1× MEM nonessential amino acids (MEM NEAA), 1× GlutaMAX (Thermo Fisher) and 2 mg/ml heparin (Sigma)] for 5 days. On day 6 (D6), 1.5 nM Bone Morphogenetic Protein (BMP4, R\&D Systems) was added to fresh NIM. On d7, EBs were plated on Matrigel. Half of the media was replaced with fresh NIM on D9, d12, and d15. On D16, the media was changed to retinal differentiation medium (RDM;DMEM:F12 3:1, 2\% B27 supplement, MEM NEAA, $1 \times$ antibiotic, antimycotic (Thermo Fisher) and $1 \times$ GlutaMAX). Retinal organoids that display an outer rim of neural retina were identified morphologically by light microscopy and dissected with a MSP ophthalmic surgical knife (Surgical Specialties Corporation) between D25-D30. Organoids were maintained in poly-HEMA-coated flasks (polyHEMA from Sigma) with twice-weekly feeding of 3D-RDM (DMEM:F12 3:1, 2\% B27 supplement, 1× MEM NEAA, 1× antibiotic, anti-mycotic, and 1× GlutaMAX with 5\% FBS, $100 \mu \mathrm{M}$ taurine, 1:1000 chemically defined lipid supplement (11905031, Thermo Fisher)). All-trans retinoic acid (1 $\mu \mathrm{M}$; Sigma) was included in the media until D120. 


\section{Quantitative real-time polymerase chain reaction (RT-qPCR)}

Gene expression was assessed by quantitative RT-qPCR. Retinal organoids (n=3-4) for each time point were homogenized using a Dounce Tissue Grinder (Sigma-Aldrich, UK) and processed using SYBR ${ }^{\text {TM }}$ Green Fast Advanced Cells-to-CT TM Kit (Invitrogen) to make cDNA. RT-qPCR was performed in triplicate using a CFX96 Real-Time System (Bio-Rad). Each primer (Table 1) was used at a final concentration of $1 \mu \mathrm{M}$. The reaction parameters were as follows: $50^{\circ} \mathrm{C}$ for 2 minutes, $95^{\circ} \mathrm{C}$ for 10 minutes to denature the cDNA and primers, 40 cycles of $95^{\circ} \mathrm{C}$ for 3 seconds followed by primer specific annealing temperature for 30 seconds $\left(60^{\circ} \mathrm{C}\right)$, succeeded by a melt curve. A comparative cycle threshold $(\mathrm{Ct})^{20}$ method was used to calculate the levels of expression that were normalized to GAPDH and relative to 0.

\section{Immunohistochemistry (IHC)}

Retinal organoids were fixed in 4\% paraformaldehyde (FD neuroTechnologies) at room temperature (RT) with gentle agitation for 35-60 minutes and washed 3x with PBS. Subsequently, retinal organoids were incubated in $15 \%$ sucrose in PBS for 1-2 hours, transferred to $30 \%$ sucrose, and stored at $4^{\circ} \mathrm{C}$ overnight. Retinal organoids were embedded in optimal cutting temperature compound and frozen at $-20^{\circ} \mathrm{C}$. Tenmicrometre cryostat sections were collected using a Leica cryostat onto Superfrost Plus slides and stored at $20^{\circ} \mathrm{C}$ in slide boxes prior to immunostaining. Cryosections were air-dried, washed several times in PBS and incubated in blocking solution (10\% normal donkey serum (NDS), 5\% bovine serum albumin, 1\% fish gelatin and $0.5 \%$ Triton X-100) for 1-2 hours at RT. Primary antibodies were incubated at $4^{\circ} \mathrm{C}$ overnight. See Table 2 for a list of primary antibodies, sources and concentrations. Secondary antibodies were diluted to 1:500 and added to tissues for 30 minutes in the dark at RT (Alexa Fluor 488, AF546 and AF647; Thermo Fisher) and again washed with PBS $(3 \times 10$ minutes). Samples were incubated in DAPI (1:1000, Thermo Fisher Scientific) for 5 minutes, and then washed with PBS $(3 \times 10$ minutes). Cover slips were mounted over the glass slides, then dried at RT and stored at $4{ }^{\circ} \mathrm{C}$ for microscopic observation. Samples were imaged on an Olympus FV1200 confocal microscope. 


\section{I-DOPA treatment}

I-DOPA was added to the media (1mM, Sigma Aldrich) with retinal organoids on D29 and removed after $12 \mathrm{~h}$ by changing the media. 3-5 retinal organoids were collected at different time points and assessed by RTqPCR described above. To avoid neurotoxicity from excess I-DOPA or DA, we therefore administered I-DOPA at lower concentration $(500 \mu \mathrm{M})$ and reduced incubation time (4 hours) for D62 retinal organoids; retinal organoids were collected every 6hours during 48hours and processed as mentioned above.

\section{Results}

To determine the appearance of dopaminergic cells, $T H$ (rate-limiting enzyme in DA biosynthesis ${ }^{21,22}$ ) mRNA was quantified on different developmental days. Retinal organoids were harvested at the same clock time (CT; 8am) each day. TH started to develop D42, was greatest D63 and decreased by D70 (Fig. 2A). At the onset of TH expression (D42), these retinal organoids were comprised of proliferating (LHX2, Ki67) neural progenitors (PAX6) and cells committed to the retinal ganglion cells (RGC) lineage (BRN3, Fig. 2B). ${ }^{23}$

Because DA is directly involved in regulating expression of circadian rhythm gene expression, we wanted to determine whether core clock genes (BMAL1, PER2, CRY1, and ROR $\alpha$ ) were rhythmically transcribed before the birth of dopaminergic cells. Therefore, these genes were assessed over a 24-hour period when retinal organoids were at D29 starting at 0 hour. At hours 4 and 8, BMAL1 and CRY1 showed lower mRNA levels than PER2 and ROR $\alpha$ (Fig. 3A). This trend reversed at hours 16 and 24. Additionally, dopaminergic gene expression of $D R D 1, D R D 2, D D C$, and $T H$ was also evaluated in these $\mathrm{D} 29$ retinal organoids. TH was minimally detected but transcription for DDC and DRD1 was upregulated at hours 4 and 12 (Fig. 3B). DDC and DRD1 mRNA levels decreased at hour 16 when DRD2 transcription was upregulated. Next, we wanted to determine if the expression of these genes would change when D29 retinal organoids were exposed to a zeitgeber. In other words, we wanted to know if these developing retinal cells were competent for circadian entrainment. Therefore, beginning at zeitgeber time 0 (ZTO), we added 1mM I-DOPA to the retinal organoids. Transcription of the core clock genes was diminished in the presence of I-DOPA at ZT4 (Fig. 3C). However, at ZT12, CRY1 transcription was upregulated while the other genes continued to express at low levels (Fig. 3C). Dopaminergic gene expression was also modified by I-DOPA. DRD1 and DDC continued to be expressed in the presence of I-DOPA at ZT4 (orange and blue lines, respectively; Fig. 3D). Conversely, 
expression of DRD2 (gray line) was repressed by I-DOPA (Fig. 3B, D). Moreover, I-DOPA stimulated expression of TH at ZT12 (Fig. 3D, yellow line). D29-D30 retinal organoids expressed mRNA that confirmed neural induction $(P A X 6)$, eye field specification $(R X)$, photoreceptor lineage $(C R X)$, and anterior neural specification (OTX2). There was an absence of mature cone photoreceptors (OPSIN) and rod precursors (NRL; Fig. 3E). Therefore, the retinal organoids were highly proliferative and unlikely to contain cells that had terminally differentiated at D29.

Next, we wanted to determine if retinal organoids rhythmically transcribed dopaminergic genes after the birth of dopaminergic cells, indicated by $\mathrm{TH}$ expression. Therefore, at D64, retinal organoids were analyzed over 48 hours for expression of DRD1, DRD2, DDC, and TH. At CT24 and CT48, TH was upregulated (Fig. 4A). Expression of DRD2 increased at CT30 (Fig. 4A). DDC was transcribed at the same times (CT24, CT30, and CT48). Assessment of BMAL1 and ROR $\alpha$ revealed maximal expression at CT 24 and CT30, respectively (Fig. 4B).

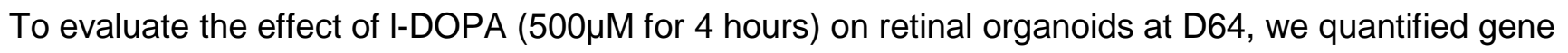
expression of the dopaminergic phenotype. mRNA expression of $D R D 2, D D C$, and $T H$ were synchronized to express maximally at ZT24 whereas DRD1 showed a delay in expression that peaked at ZT30 (Fig. 4C). Expression of BMAL1 was antiphase to ROR $\alpha$ after I-DOPA treatment (Fig. 4D). IHC at D62 indicated the presence of RGCs (SNCG $/ \mathrm{CaR}^{-}$, green arrow and $\mathrm{SNCG}^{+} / \mathrm{CaR}^{+}$, white arrow), amacrine cells (SNCG $/ \mathrm{CaR}^{+}$, red arrow; Fig. 4E) and photoreceptor progenitors (CRX and OTX2, red arrow; Fig. 4F). Differentiated cells at this developmental stage was also supported by minimal detection mitotic proliferation (Ki67; Fig. 4G). Therefore, D64 retinal organoids consisted mainly of terminally differentiated inner retinal cells.

\section{Discussion}

Here we show that DA signaling genes (DRD1, DRD2, $T H, D D C)$ begin to express autonomously around D42-D70, without extrinsic I-DOPA. Furthermore, in the proliferating retinal cells, extrinsic I-DOPA triggered mRNA expression of DA signaling genes. This has also been reported in chick retinae. Kubrusly et a/ ${ }^{15}$ showed that the retinal pigmented epithelium (RPE) supplies I-DOPA prior to the birth of dopaminergic cells which stimulates DA synthesis. ${ }^{15}$ However, whether I-DOPA is released from human RPE during development has yet to be determined. 
It has been suggested that the clock could play a major role in determining the timing of cell differentiation during development. ${ }^{24}$ Recently, core clock proteins have been postulated to restrict oscillations in undifferentiated cells ${ }^{25}$ but upon differentiation, may guide neuron migration and synaptic network formation. ${ }^{26}$ In vivo and in synchronized cells, PER1/2 and CRY1/2 are upregulated during the subjective day so that they can form a complex that subsequently negatively regulate the expression of clock genes. ${ }^{27-29}$ We did not find parallel expression of CRY1 and PER2 at D29 and I-DOPA did not synchronize mRNA expression of these genes (Fig. 3). Additionally, the retinoic acid-related orphan receptor (RORa, $\beta, \mathrm{c})$ protein is known to stimulate gene expression of BMAL1 which is occurs during the subjective night. ${ }^{3,30-32}$ However, we did not find BMAL1 transcription to be increased following the upregulation of ROR $\alpha \mathrm{mRNA}$ expression at D29. Again, I-DOPA did not stimulate ROR $\alpha$ mRNA expression nor subsequent BMAL1 transcription at D29. Conversely, CRY1 expression was upregulated by I-DOPA. It has been suggested that during development, CRY1 has regulatory functions that are independent of the circadian transcription/translation feedback loop that is active in almost every cell in adults. ${ }^{33}$ As such, I-DOPA may be required for CRY1 expression during embryogenesis. The D29 retinal organoids were comprised of undifferentiated and proliferating progenitor cells. Altogether, these results support the notion that the role of circadian mechanisms in early retinogenesis (before cells terminally differentiate) is to restrict oscillations and prevent synchronization of circadian gene expression.

At D29, the retinal organoids were highly proliferative and at a stage of development prior to inner retinal cell differentiation. We do not know if expression of $T H$, albeit was extremely low, had functional significance. To determine whether DA is synthesized and secreted from proliferating progenitors as well as from DACs, we need to evaluate DA (mass spectrometry) in media and retinal organoids at $\sim$ D29 and $\sim$ D64, respectively. Nevertheless, even with the low levels of $T H$, D29 retinal organoids expressed $D D C, D R D 1$ and DRD2. Specifically, expression of DRD1 mRNA was higher when DRD2 showed lower expression. Conversely, when transcription of DRD1 was decreased, DRD2 mRNA increased in D29 retinal organoids (Fig. 3). On the other hand, D62 retinal organoids showed a preference for DRD2 mRNA expression and not DRD1 (Fig. 4A and 3C). Moreover, D62 retinal organoids responded differently to I-DOPA treatment compared to D29. At this stage, I-DOPA did not directly stimulate the expression of DRD1, but instead, acted as a zeitgeber by phase shifting the expression of DRD2, BMAL1, and ROR $\alpha$ (Fig. 4). Unlike D29, at D64, transcription of BMAL1 and ROR $\alpha$ was set by I-DOPA where increased ROR $\alpha$ mRNA expression occurred 
when BMAL1 was downregulated. Conversely, when BMAL1 increased mRNA expression, ROR $\alpha$ was downregulated (Fig. 4D). It is uncertain whether a single treatment with I-DOPA will be sufficient to synchronize cyclic dopaminergic gene expression in retinal organoids. In order to establish rhythmicity, these measures need to be assessed for at least 5 days including time points throughout each day. ${ }^{34}$ Altogether, results from this study show unique DA signaling at different stages of development in the human retina. This suggests that the specific pathways triggered by DA mediates distinct physiological processes during retinal neurogenesis that varies among proliferating, differentiating, and terminally differentiated retinal neurons. 


\section{References:}

1. Besharse JC, McMahon DG. The Retina and Other Light-sensitive Ocular Clocks. J Biol Rhythms 2016;31:223-243.

2. $\quad$ Liang FQ, Green L, Wang C, Alssadi R, Godley BF. Melatonin protects human retinal pigment epithelial (RPE) cells against oxidative stress. Experimental eye research 2004;78:1069-1075.

3. Tosini G, Pozdeyev N, Sakamoto K, Iuvone PM. The circadian clock system in the mammalian retina. BioEssays : news and reviews in molecular, cellular and developmental biology 2008;30:624-633.

4. Stone RA, Lin T, Laties AM, Iuvone PM. Retinal dopamine and form-deprivation myopia. Proceedings of the National Academy of Sciences of the United States of America 1989;86:704-706.

5. Girault JA, Greengard P. The neurobiology of dopamine signaling. Arch Neurol 2004;61:641-644.

6. Neve KA, Seamans JK, Trantham-Davidson H. Dopamine receptor signaling. J Recept Signal Transduct Res 2004;24:165-205.

7. Seamans JK, Yang CR. The principal features and mechanisms of dopamine modulation in the prefrontal cortex. Prog Neurobiol 2004;74:1-58.

8. Brandies R, Yehuda S. The possible role of retinal dopaminergic system in visual performance. Neurosci Biobehav Rev 2008;32:611-656.

9. Shimada H, Lu Q, Insinna-Kettenhofen C, et al. In Vitro Modeling Using Ciliopathy-Patient-Derived Cells Reveals Distinct Cilia Dysfunctions Caused by CEP290 Mutations. Cell Rep 2017;20:384-396.

10. Popolo M, McCarthy DM, Bhide PG. Influence of dopamine on precursor cell proliferation and differentiation in the embryonic mouse telencephalon. Dev Neurosci 2004;26:229-244.

11. Crandall JE, McCarthy DM, Araki KY, Sims JR, Ren JQ, Bhide PG. Dopamine receptor activation modulates GABA neuron migration from the basal forebrain to the cerebral cortex. J Neurosci 2007;27:38133822.

12. Todd RD. Neural development is regulated by classical neurotransmitters: dopamine D2 receptor stimulation enhances neurite outgrowth. Biol Psychiatry 1992;31:794-807.

13. Reinoso BS, Undie AS, Levitt P. Dopamine receptors mediate differential morphological effects on cerebral cortical neurons in vitro. Journal of neuroscience research 1996;43:439-453.

14. Tojima T, Kobayashi S, Ito E. Dual role of cyclic AMP-dependent protein kinase in neuritogenesis and synaptogenesis during neuronal differentiation. Journal of neuroscience research 2003;74:829-837.

15. Kubrusly RC, Guimaraes MZ, Vieira AP, et al. L-DOPA supply to the neuro retina activates dopaminergic communication at the early stages of embryonic development. Journal of neurochemistry 2003;86:45-54.

16. Lankford KL, DeMello FG, Klein WL. D1-type dopamine receptors inhibit growth cone motility in cultured retina neurons: evidence that neurotransmitters act as morphogenic growth regulators in the developing central nervous system. Proceedings of the National Academy of Sciences of the United States of America 1988;85:4567-4571.

17. Guimaraes MZ, Hokoc JN, Duvoisin R, Reis RA, De Mello FG. Dopaminergic retinal cell differentiation in culture: modulation by forskolin and dopamine. Eur J Neurosci 2001;13:1931-1937.

18. Meyer JS, Shearer RL, Capowski EE, et al. Modeling early retinal development with human embryonic and induced pluripotent stem cells. Proceedings of the National Academy of Sciences of the United States of America 2009;106:16698-16703.

19. Meyer JS, Howden SE, Wallace KA, et al. Optic vesicle-like structures derived from human pluripotent stem cells facilitate a customized approach to retinal disease treatment. Stem Cells 2011;29:1206-1218.

20. Livak KJ, Schmittgen TD. Analysis of relative gene expression data using real-time quantitative PCR and the 2(-Delta Delta C(T)) Method. Methods 2001;25:402-408.

21. Nagatsu T. Tyrosine hydroxylase: human isoforms, structure and regulation in physiology and pathology. Essays Biochem 1995;30:15-35.

22. Kaufman S. Tyrosine hydroxylase. Adv Enzymol Relat Areas Mol Biol 1995;70:103-220. 
23. Reichman S, Terray A, Slembrouck A, et al. From confluent human iPS cells to self-forming neural retina and retinal pigmented epithelium. Proceedings of the National Academy of Sciences of the United States of America 2014;111:8518-8523.

24. Froland Steindal IA, Whitmore D. Circadian Clocks in Fish-What Have We Learned so far? Biology (Basel) 2019;8.

25. Umemura Y, Maki I, Tsuchiya Y, Koike N, Yagita K. Human Circadian Molecular Oscillation Development Using Induced Pluripotent Stem Cells. J Biol Rhythms 2019;34:525-532.

26. Noda M, Iwamoto I, Tabata H, Yamagata T, Ito H, Nagata KI. Role of Per3, a circadian clock gene, in embryonic development of mouse cerebral cortex. Sci Rep 2019;9:5874.

27. McMahon DG, Iuvone PM, Tosini G. Circadian organization of the mammalian retina: from gene regulation to physiology and diseases. Progress in retinal and eye research 2014;39:58-76.

28. Mohawk JA, Green CB, Takahashi JS. Central and peripheral circadian clocks in mammals. Annu Rev Neurosci 2012;35:445-462.

29. Partch CL, Green CB, Takahashi JS. Molecular architecture of the mammalian circadian clock. Trends Cell Biol 2014;24:90-99.

30. Preitner N, Damiola F, Lopez-Molina L, et al. The orphan nuclear receptor REV-ERBalpha controls circadian transcription within the positive limb of the mammalian circadian oscillator. Cell 2002;110:251-260. 31. Hogenesch JB, Ueda HR. Understanding systems-level properties: timely stories from the study of clocks. Nat Rev Genet 2011;12:407-416.

32. Ukai-Tadenuma M, Yamada RG, Xu H, Ripperger JA, Liu AC, Ueda HR. Delay in feedback repression by cryptochrome 1 is required for circadian clock function. Cell 2011;144:268-281.

33. Amano T, Matsushita A, Hatanaka Y, et al. Expression and functional analyses of circadian genes in mouse oocytes and preimplantation embryos: Cry1 is involved in the meiotic process independently of circadian clock regulation. Biol Reprod 2009;80:473-483.

34. Refinetti R, Lissen GC, Halberg F. Procedures for numerical analysis of circadian rhythms. Biol Rhythm Res 2007;38:275-325. 
bioRxiv preprint doi: https://doi.org/10.1101/2020.10.14.339366; this version posted October 14, 2020. The copyright holder for this preprint (which was not certified by peer review) is the author/funder. All rights reserved. No reuse allowed without permission.

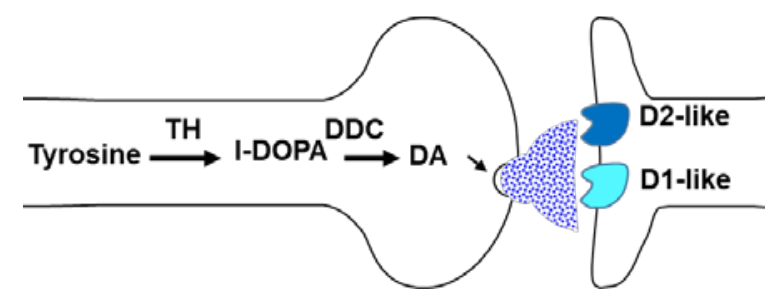

Figure 1. Dopamine biosynthesis. 
bioRxiv preprint doi: https://doi.org/10.1101/2020.10.14.339366; this version posted October 14, 2020. The copyright holder for this preprint (which was not certified by peer review) is the author/funder. All rights reserved. No reuse allowed without permission.

A.

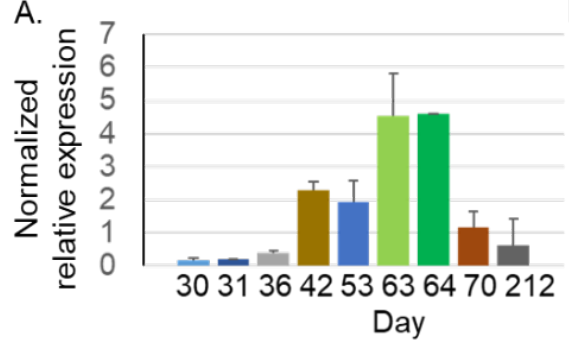

B.

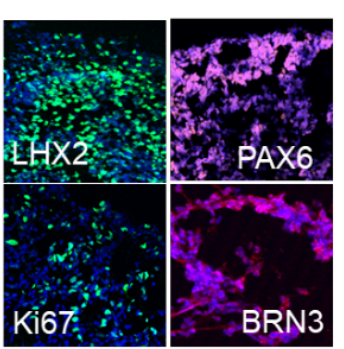

Figure 2 A) D30-D212 TH mRNA \pm SD normalized (GAPDH); triplicates. $n=3-4$ B) IHC of proliferating (LHX2, PAX6, Ki67) and RGC-committed cells (BRN3). 


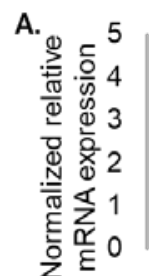

B.

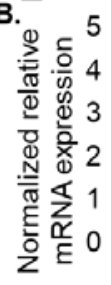

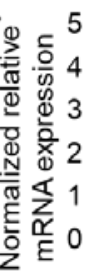

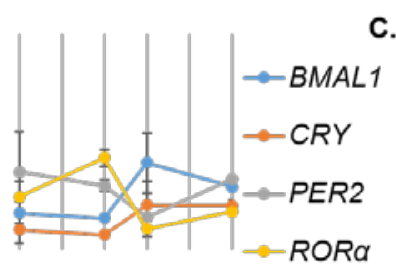

c.

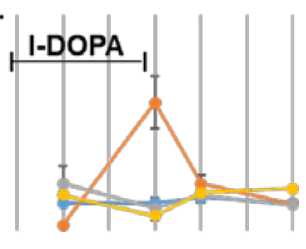

D.

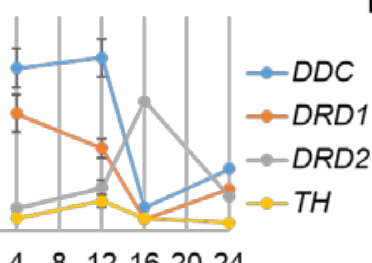

$0 \quad 4 \quad 812162024$

Hour

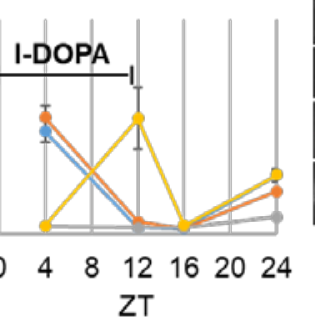

E.

$0^{6} 0^{1} 0^{3^{8}}$

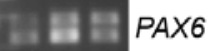

at $R X$

$\because C R X$

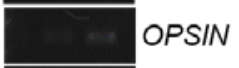

$N R L$

Пอ ОТХ2

GAPDH

Figure 3 A) D29 retinal organoids mRNA expression of core clock and $\mathbf{B}$ ) dopaminergic genes $\mathbf{C}$ ) I-DOPA changed the pattern of gene expression of clock genes and D) downregulated DRD2. ZT, zeitgeber time (hours) E) RT-PCR showing neural progenitors. 


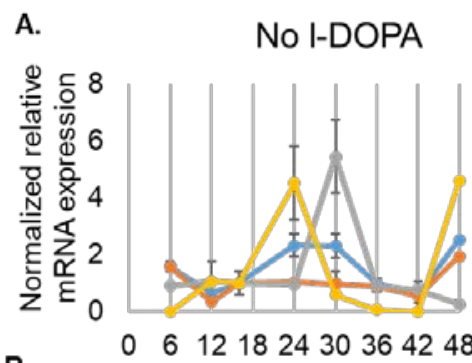

B.

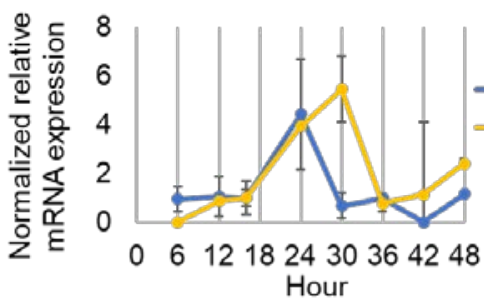

$0 \quad 612182430364248$
C. + I-DOPA

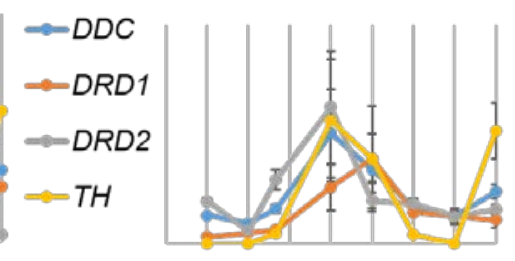

D.
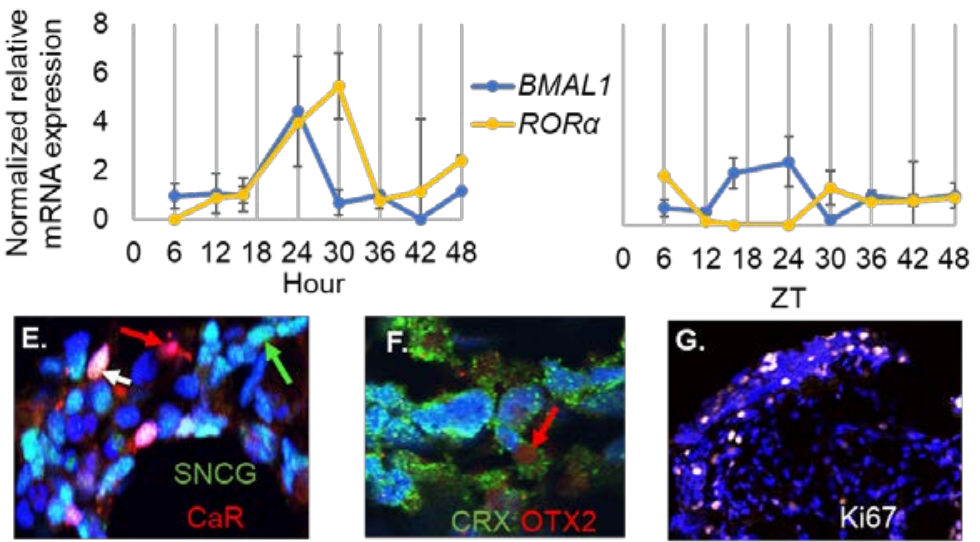

$\mathrm{ZT}$

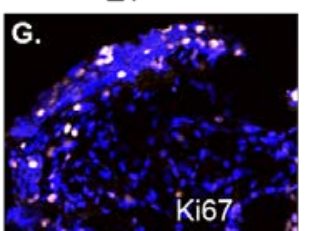

Figure 4. D62 retinal organoids. mRNA expression A), B), C) and D) 4 hours incubation I-DOPA, ZT, zeitgeber time (hours). E) IHC D62 showing SNCG (RGCs, green arrow), CaR (RGC, white arrow; amacrine cell, red arrow). F) Photoreceptor progenitors CRX and OTX2 (red arrow). G) Minimal mitotic proliferation (Ki67) 


\section{Table 1}

\begin{tabular}{|c|c|c|}
\hline Gene & Forward Primer & Reverse Primer \\
\hline$B M A L 1$ & GAC TTC CCC TCT ACC TGC TC & AAT AGC TGT TGC CCT CTG GT \\
\hline CRX & $\begin{array}{l}\text { TGA TGC ACC AGG CTG TGC CCT } \\
\text { A }\end{array}$ & TGG CAA ACA GTG CCT CCA GCT C \\
\hline CRY1 & GCC GTT TTG TCT TTA GGG GC & ACA CTG AAG CAA AAA TCG CCA \\
\hline$D D C$ & ATGCACAGAGCTGGAGACTG & CACTTCCCTGGATCACTCCT \\
\hline DRD1 & AGCGAAGTCCACATTCCAAG & ATTCTCCCCTTCTGAGACTCA \\
\hline DRD2 & GTCATGATCTCCATCGTCTGG & AATGAAGGGCACGTAGAAGG \\
\hline GAPDH & ACC ACA GTC CAT GCC AT CAC & TCC ACC ACC CTG TTG CTG TA \\
\hline NRL & $\begin{array}{l}\text { AGT TTG AGG TAA AGC GGG AAC } \\
C C T\end{array}$ & ACC ATG CCT GGT TCA CTGAAG GT \\
\hline OPSIN & $\begin{array}{l}\text { TAC CTG GAC CAT TGG TAT TGG } \\
\text { CGT }\end{array}$ & TAA GTC CAG CCC ATG GTT ACG GTT \\
\hline OTX2 & AGA GCA GCC CTC ACT CGC CA & AGT CGG CCC AAA TCG GGG GT \\
\hline PAX6 & $\begin{array}{l}\text { AGT GAA TCA GCT CGG TGG TGT } \\
\text { CTT }\end{array}$ & $\begin{array}{l}\text { TGC AGA ATT CGG GAA ATG TCG } \\
\text { CAC }\end{array}$ \\
\hline PER2 & TGG ACT CCT CGG CTT GAA AC & AAG GTA TCT GGA CTC TGG CG \\
\hline$R X$ & $\begin{array}{l}\text { AGC GAA ACT GTC AGA GGA GGA } \\
\text { ACA }\end{array}$ & $\begin{array}{l}\text { TCA TGC AGC TGG TAC GTG GTG } \\
A A A\end{array}$ \\
\hline$R O R \alpha$ & ACG CCС АCС ТАC АAC АТC ТC & CAC AAT TGC CAC ATC АCC TC \\
\hline $\mathrm{TH}$ & $\begin{array}{l}\text { GAG ACG TTT GAA GCC AAA ATC } \\
\text { C }\end{array}$ & AGG TCA GGG TCG AAC TTG GT \\
\hline
\end{tabular}


bioRxiv preprint doi: https://doi.org/10.1101/2020.10.14.339366; this version posted October 14, 2020. The copyright holder for this preprint (which was not certified by peer review) is the author/funder. All rights reserved. No reuse allowed without permission.

Table 2

\begin{tabular}{|l|l|l|l|l|}
\hline Antibodies & type & source & Cat number & Dilution \\
\hline BRN3 & $\begin{array}{l}\text { Rabbit } \\
\text { polyclonal }\end{array}$ & Thermofisher & PA541509 & $1: 50$ \\
\hline CaR & $\begin{array}{l}\text { Rabbit } \\
\text { polyclonal }\end{array}$ & Milipore & AB5054 & $1: 500$ \\
\hline CRX & $\begin{array}{l}\text { Mouse } \\
\text { monoclonal }\end{array}$ & Abnova & $\begin{array}{l}\text { H00001406- } \\
\text { M02 }\end{array}$ & $1: 100$ \\
\hline Ki-67 & $\begin{array}{l}\text { Mouse } \\
\text { monoclonal }\end{array}$ & $\begin{array}{l}\text { BD } \\
\text { Biosciences }\end{array}$ & 556003 & $1: 500$ \\
\hline LHX2 & $\begin{array}{l}\text { Mouse } \\
\text { monoclonal }\end{array}$ & Santa Cruz & Sc-81311 & $1: 50$ \\
\hline OTX2 & $\begin{array}{l}\text { Goat } \\
\text { polyclonal }\end{array}$ & R\&D & AF1979 & $1: 2000$ \\
\hline PAX6 & $\begin{array}{l}\text { Mouse } \\
\text { monoclonal }\end{array}$ & DSHB & Pax6-s & $1: 50$ \\
\hline SNCG & $\begin{array}{l}\text { Mouse } \\
\text { monoclonal }\end{array}$ & Abnova & $\begin{array}{l}\text { H00006623- } \\
\text { M01 }\end{array}$ & $1: 500$ \\
\hline
\end{tabular}

